\title{
Anticholinergic Activity and Alzheimer's Disease
}

\author{
Koji Hori $^{\mathrm{a}}$ Mitsugu Hachisu ${ }^{c}$ Hiroi Tomioka ${ }^{a}$ Kimiko Konishi ${ }^{a}{ }^{a}$ \\ ${ }^{a}$ Department of Psychiatry, Showa University Northern Yokohama Hospital, Yokohama, and ${ }^{\text {b}}$ Tokyo Metropolitan \\ Tobu Medical Center for Persons with Developmental/Multiple Disabilities and ' Department of Pharmaceutical \\ Therapeutics, Division of Clinical Pharmacy, School of Pharmacy, Showa University, Tokyo, Japan
}

As members of the Showa University Dementia Study Group (Wade Study Group), we greatly appreciate the opportunities we have to write about the relationship between anticholinergic activity (AA) and neuropsychiatric disorders, and Alzheimer's disease (AD) in particular. In this special issue, we review our previous articles concerning the endogenous hypothesis of $\mathrm{AA}$ in $\mathrm{AD}$ that we originally proposed and here extend the hypothesis to various neurocognitive disorders such as Lewy body disease (LBD), delirium and neuropsychiatric disorders including depression and schizophrenia.

In this issue, we emphasize the following 3 points with regard to our hypothesis. First, AA not only depresses the acetylcholine $(\mathrm{ACh})$ system through antagonization of ACh receptors, but also accelerates the accumulation of insoluble amyloids in the brain. The resulting dysfunction of the ACh system is not always reversible. Second, downregulation of ACh can also induce AA by way of inflammation. Therefore, the relationships between AA and this dysfunction of the ACh system are bidirectional. Given that downregulation of the ACh system is an essential feature of $\mathrm{AD}$, we consider that endogenous $\mathrm{AA}$ accelerates $\mathrm{AD}$ pathology. We refer to these bidirectional effects of $\mathrm{AA}$ and downregulation of $\mathrm{ACh}$ as either the 'hypothesis of endogenous $\mathrm{AA}$ in $\mathrm{AD}$ ', 'the endogenous
AA cascade in $\mathrm{AD}$ ' or 'progression of $\mathrm{AD}$ by its own mechanism'. Although there are AA-inducing factors other than downregulation of ACh (e.g. drugs, febrile illness or mental stress), upregulation of $\mathrm{ACh}$ is possible if the ACh system is not depressed or overloaded. Thus, upregulation of the ACh system can inhibit $\mathrm{AA}$ even in the presence of other factors (fig. 1). The appearance of AA would then suggest that the ACh system is impaired in some way or that disease other than $\mathrm{AD}$ is responsible for the AA.

Third, downregulation of ACh in the central nervous system (CNS) causes upregulation of inflammation in the CNS and other organ systems. It is, therefore, conceivable that $\mathrm{AD}$, a disease of the CNS, and diabetes mellitus, a disease that affects the peripheral nervous system, have some aspects of pathogenesis in common. Moreover, downregulation of the ACh system seems to be responsible for the simultaneous appearance of AA in the CNS and other organ systems. A correlation between AA in both systems can be explained, then, not only by the permeability of AA through the blood-brain barrier, but also by this simultaneous appearance of AA in both systems.

Dr. Tomioka, MD, PhD, Dr. Hachisu, PhD, and Dr. Hori, $\mathrm{MD}, \mathrm{PhD}$, conceived the contents of this special issue. We are extremely grateful to all authors for their

\section{KARGER 125}

(c) 2015 S. Karger AG, Base

$1660-2854 / 15 / 0153-0131 \$ 39.50 / 0$
Koji Hori, MD, PhD

Department of Psychiatry, Showa University Northern Yokohama Hospital 35-1 Chigasakichuo, Tsuzukiku

Yokohama, Kanagawa 224-8503 (Japan)

E-Mail kojihori@med.showa-u.ac.jp 


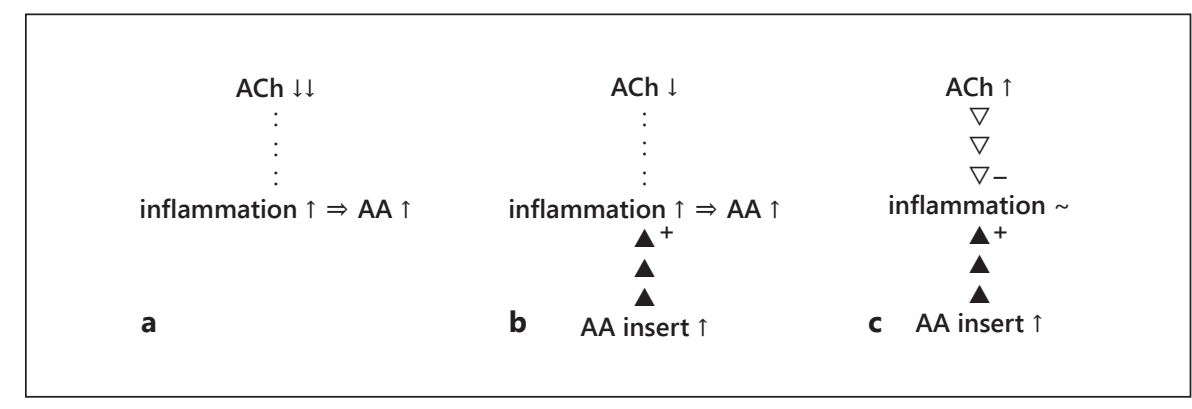

Fig. 1. a We speculate that when downregulation of ACh reaches a critical level, the inflammatory system is upregulated and cytokines that have AA appear. b Even if downregulation of ACh does not reach a critical level, when another AA-inducing factor is present (e.g. medication, febrile illness or mental stress), the inflamma- tory system is upregulated, and AA appears. c When the ACh system is intact and not depressed or overloaded, upregulation of $\mathrm{ACh}$ is possible even if another AA-inducing factor is present (e.g. medication, febrile illness or mental stress). Consequently, the inflammatory system is not upregulated, and AA does not appear. valuable contributions to this publication: Dr. Hachisu for explaining serum AA as a peripheral biological marker of AA in the CNS; Dr. Yoshiyama, MD, PhD, for discussing the adverse effects of AA in the brain from the viewpoint of neuropathology; Dr. Konishi, $\mathrm{PhD}$, for explaining the endogenous appearance of $\mathrm{AA}$ in $\mathrm{AD}$; Dr. Kitajima, $\mathrm{MD}, \mathrm{PhD}$, for explaining AA in neurocognitive disorders other than $\mathrm{AD}$ that show downregulation of ACh; Dr. Hosoi, PhD, for discussing pharmacotherapy for $\mathrm{AD}$ and $\mathrm{LBD}$ based on the hypothesis of $\mathrm{AA}$ in $\mathrm{AD}$ and $\mathrm{LBD}$; Dr. Hori for covering $\mathrm{AA}$ in depression; Dr. Tani, MD, $\mathrm{PhD}$, for explaining AA in schizophrenia; Dr. Hosoi for covering plasma cholinesterase activity as another biological marker of $\mathrm{AD}$, and Dr. Hachisu for discussing brain-derived neurotrophic factor in AD. We additionally thank Dr. Tomioka, Dr. Hachisu, Dr. Konishi and Dr. Hori for their careful checking of the papers.

The two main aims of this special issue were to review and refine our hypothesis of the endogenous appearance of AA in AD (article by Dr. Konishi) and to demonstrate the utility of serum AA for evaluating the anticholinergic burden in the CNS (article by Dr. Hachisu). The refined hypothesis was derived from the findings presented in our 3 previous reports [1-3] and the review process we undertook. Therefore, it is a limitation of this special issue that our discussion is derived mainly from reviewing our 2 original articles [4, 5], 3 case reports [6-8] and review articles [1-3] whose findings have not been proven in large-scale studies. We have presented our refined hypothesis in just 1 article [9], and this special issue marks the first publication of our additional suggestions, implications and case presentations supporting our revised hy- pothesis. Moreover, through this reviewing of our hypothesis, we have extended it to cover other neurocognitive disorders, such as LBD and delirium, and other neuropsychiatric disorders, such as depression and schizophrenia. We have also proposed other biological markers for $\mathrm{AD}$, including plasma cholinesterase and brain-derived neurotrophic factor. In the future, we hope to prove our hypothesis in clinical settings.

As a final note, we designed this special issue so that the articles could be read in any order and we ask the readers' indulgence of some repetition of our endogenous hypothesis of $\mathrm{AA}$ in $\mathrm{AD}$ at the beginning of certain articles to allow for this. We sincerely hope that this special issue is of value to our readers.

\section{Acknowledgments}

Funding for this study was received from Eisai Co. Ltd., Daiichi Sankyo Inc. and Ono Pharmaceutical Co. Ltd.

We thank Karger EDA for their great assistance.

\section{Disclosure Statement}

Koji Hori has received lecture fees from Eisai Co. Ltd., Pfizer Japan Inc., Novartis Pharma KK, Daiichi Sankyo Inc., Ono Pharmacuetical Co. Ltd., Janssen Pharmaceutical KK, Yoshitomi Yakuhin Co. and Mitsubishi Tanabe Pharma Co. Mitsugu Hachisu has received funding from Astellas Pharma Inc., Meiji Seika Pharma Co. Ltd., Dainippon Sumitomo Pharm Co. Ltd., Eli Lilly Japan KK and Shionogi \& Co. Ltd., and received lecture fees from Meiji Seika Pharma Co. Ltd. and Mitsubishi Tanabe Pharma Co. 


\section{References}

-1 Hori K, Konishi K, Akita R, Tani M, Tomioka H, Kitajima Y, Yokoyama S, Azuma K, Ikuse D, Akashi N, Yuda H, Hachisu M: Proposal of endogenous anticholinergic hypothesis in Alzheimer's disease. Jpn J Neuropsychopharmacol 2013;33:117-126.

-2 Hori K, Konishi K, Tani M, Tomioka H, Akita R, Kitajima Y, Aoki M, Yokoyama S, Azuma K, Ikuse D, Akashi N, Hosoi M, Jinbo K, Hachisu M: Serum anticholinergic activity: a possible peripheral marker of the anticholinergic burden in the central nervous system in Alzheimer's disease. Dis Markers 2014;2014: 459013.

3 Hori K, Konishi K, Tani M, Tomioka H, Akita R, Kitajima Y, Aoki M, Kikuchi N, Ikuse D, Akashi N, Hosoi M, Jinbo K, Hachisu M: Why does the progression of Alzheimer's disease accelerate? Ann Psychiatry Ment Health 2014;2:1006.
4 Konishi K, Hori K, Uchida H, Watanabe K, Tominaga I, Kimura M, Hosoyamada M, Shibasaki T, Kataoka A, Hachisu M: Adverse effects of anticholinergic activity on cognitive functions in Alzheimer's disease. Psychogeriatrics 2010;10:34-38.

5 Hori K, Konishi K, Watanabe K, Uchida H, Tsuboi T, Moriyasu M, Tominaga I, Hachisu $\mathrm{M}$ : Influence of anti-cholinergic activity in serum on clinical symptoms of Alzheimer's disease. Neuropsychobiology 2011;63:147-153.

6 Hori K, Funaba Y, Konishi K, Moriyasu M, Hirata K, Oyamada R, Tominaga I, Inada T: Assessment of pharmacological toxicity using serum anticholinergic activity in a patient with dementia. Psychiatry Clin Neurosci 2005;59:508-510.
7 Hori K, Konishi K, Minegishi G, Tomioka H, Tani M, Tanaka H, Akita R, Yokoyama S. Oshio T, Hachisu M: Memantine abolishes anticholinergic activity in patient with Alzheimer's disease at moderate stage. J Alzheimer Dis Parkinsonism 2012;2:3.

$>$ Konishi K, Hori K, Tomioka H, Minegishi G, Tani M, Tanaka H, Akita R, Yokoyama S, Oshio T, Hachisu M: Donepezil abolishes anticholinergic activity in a patient with amnesia. Pharmacology 2013;91:86-91.

9 Kitajima Y, Yamaguchi K, Hori K, Murakami T, Someya A, Konishi K, Hara A, Hachisu M, Kajiyama Y, Nagaoka I, Inada E: The relationship between the perioperative transition of serum anticholinergic activity and postoperative delirium in patients undergoing esophagectomy and gastrectomy. Juntendo Med J 2014;60:147-150. 